\title{
Online MALDI-TOF MS Using an Aerodynamic Lens Assembly as a Direct Deposition Interface
}

\author{
Jinian Shu,* Junwang Meng, Xi Shu, Yang Zhang, Shaokai Gao, Bo Yang, and Jie Gan \\ Research Center for Eco-Environmental Sciences, Chinese Academy of Sciences, Beijing 100085, China
}

This technical note reports a design utilizing an aerodynamic lens assembly to transport suspended sample particles directly onto the target plate and conduct in situ MALDI analysis. The design provides a new alternative method for online MALDI analysis. The matrix/analyte/ salt-contained particles sampled by a nozzle are focused into a particle beam with an aerodynamic lens assembly and then deposited on the target plate directly. The deposited particles are in situ desorbed/ionized by a 266 $\mathrm{nm}$ laser beam with an incidence angle of 50 degrees. The generated ions are detected with a reflectron mass spectrometer capable of delayed ion extraction and ion gating. The MALDI-TOF mass spectra of poly(ethylene glycol) (PEG) 1000, 2000, and 4000 particles are obtained in the experiment. The initial experimental results demonstrate that the design can be used for online MALDI analysis.

Matrix-assisted laser desorption/ionization time-of-flight mass spectrometry (MALDI MS) has been widely used since its introduction in the late 1980s. The soft ionization technique of MALDI is one of the methods that enable acquisition of the mass spectra of large molecules such as biomolecules, synthetic polymers, carbohydrates, etc. ${ }^{1-4}$ Most commercial MALDI MS is used to analyze the solid sample offline. The samples are prepared by depositing the solution containing matrix and analyte on the target plate. The solution forms crystal film on the target plate after the solvent vaporizes.

Many attempts have been made to realize online MALDI analysis. Coupling a capillary directly to the MALDI sample probe was an approach to make online MALDI analysis. Using the method, MALDI MS can be combined with liquid separation devices such as liquid chromatography and capillary electrophoresis for combinative analysis. ${ }^{5,6}$ Using a rotating ball (or a wheel) inlet was also an alternative for online MALDI MS. The rotating ball continuously picked up the sample delivered from a capillary by surface adherence, and then, a laser illuminated the ball surface

* Corresponding author. Phone: +86 0106284 9508. Fax: +86 01062923563. E-mail: jshu@rcees.ac.cn.

(1) Yates, J. R. J. Mass Spectrom. 1998, 33 (1), 1-19.

(2) Montaudo, G.; Samperi, F.; Montaudo, M. S. Prog. Polym. Sci. 2006, 31 (3), 277-357.

(3) Nielen, M. W. F. Mass Spectrom. Rev. 1999, 18 (5), 309-344.

(4) Harvey, D. J. Mass Spectrom. Rev. 1999, 18 (6), 349-450.

(5) Li, L.; Wang, A. P. L.; Coulson, L. D. Anal. Chem. 1993, 65 (4), 493-495.

(6) Zhan, Q.; Gusev, A.; Hercules, D. M. Rapid Commun. Mass Spectrom. 1999, 13 (22), 2278-2283. to desorb/ionize the sample. ${ }^{7,8}$ Aerosol-MALDI MS was a technique used by many research groups to achieve online MALDI analysis. The analyte/matrix-contained particles (droplets) or matrix-coated particles sampled by an inlet flew through the detection region of aerosol-MALDI MS continuously, where the particles were intercepted by a desorption/ionization laser. ${ }^{9-14}$ Single-particle analysis can be realized by the use of this technique. ${ }^{10}$

Recently, Gao and Johnston reported a deposition method of nanoaerosol on the MALDI target plate for MALDI analysis. ${ }^{15}$ The nanoaerosol particles were focused with an aerodynamic lens assembly forming a sample spot with hundreds of micrometers on the target plate. After the deposition, the target plate was inserted into MALDI MS for analysis. In the present technical note, we report a design of an aerodynamic lens coupled matrixassisted desorption/ionization time-of-flight mass spectrometer (AL-MALDI MS). Using an aerodynamic lens as a direct deposition interface, the design enables AL-MALDI MS to introduce suspended sample particles under atmospheric pressure continuously and conduct instant MALDI analysis. The design of ALMALDI MS and the initial results are reported in the following sections.

\section{EXPERIMENTAL SECTION}

The sectional view of AL-MALDI MS is shown in Figure 1. The suspended particles containing matrix and analyte are introduced into the detection chamber with a nozzle of $\sim 0.12 \mathrm{~mm}$ orifice combined with an aerodynamic lens assembly and a three stage differential pumping system. The nozzle orifice is custommade by Beijing Sanhejiaming Laser Technology Development Com., Ltd. (China). It is a stainless steel disk $(0.2 \mathrm{~mm}$ thick and $9.5 \mathrm{~mm}$ in diameter) with a centered microhole punched by a

(7) Preisler, J.; Foret, F.; Karger, B. L. Anal. Chem. 1998, 70 (24), 52785287.

(8) Orsnes, H.; Graf, T.; Degn, H.; Murray, K. K. Anal. Chem. 2000, 72 (1), 251-254.

(9) Murray, K. K.; Russell, D. H. Anal. Chem. 1993, 65 (18), 2534-2537.

(10) Mansoori, B. A.; Johnston, M. V.; Wexler, A. S. Anal. Chem. 1996, 68 (20), 3595-3601.

(11) Fei, X.; Wei, G.; Murray, K. K. Anal. Chem. 1996, 68 (7), 1143-1147.

(12) Stowers, M. A.; van Wuijckhuijse, A. L.; Marijnissen, J. C. M.; Scarlett, B.; van Baar, B. L. M.; Kientz, C. E. Rapid Commun. Mass Spectrom. 2000, 14 (10), 829-833.

(13) Harris, W. A.; Reilly, P. T. A.; Whitten, W. B. Int. J. Mass Spectrom. 2006, 258 (1-3), 113-119.

(14) Bogan, M. J.; Patton, E.; Srivastava, A.; Martin, S.; Fergenson, D. P.; Steele, P. T.; Tobias, H. J.; Gard, E. E.; Frank, M. Rapid Commun. Mass Spectrom. 2007, 21 (7), 1214-1220.

(15) Gao, Y. Q.; Johnston, M. V. Rapid Commun. Mass Spectrom. 2009, 23 (24), 3963-3968. 


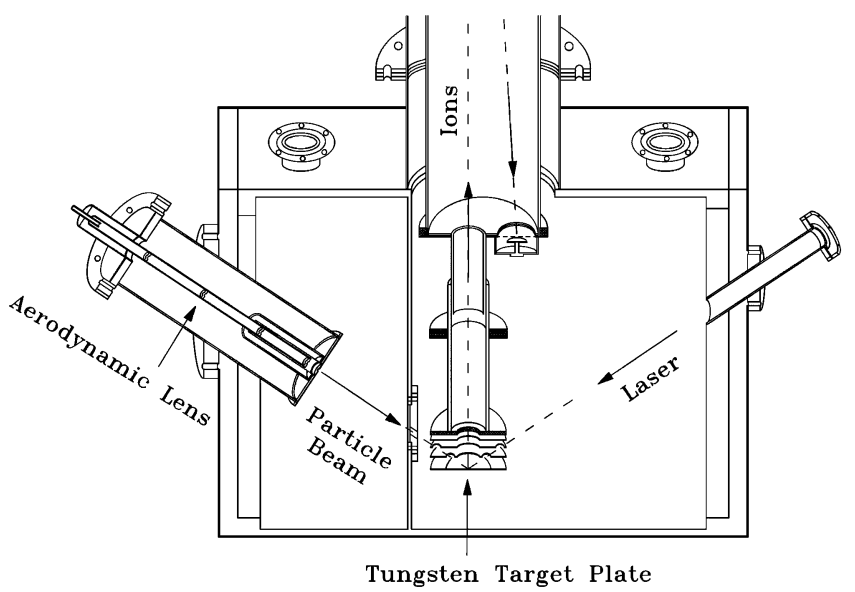

Figure 1. Sectional view of AL-MALDI MS.

laser. The sampling flow rate is $\sim 1.3 \mathrm{~cm}^{3} \mathrm{~s}^{-1}$. The aerodynamic lens assembly is machined using the design considerations reported by Zhang, ${ }^{16}$ which are capable of focusing the particles with the diameters ranging from 70 to $1000 \mathrm{~nm}$. The aerodynamic lens assembly is tilted at 40 degrees from the horizontal plane. The aerodynamic lens assembly focuses the suspended particles into a particle beam during the delivery of the particles into the vacuum. The particle beam is deposited continuously on a tungsten target plate which also serves as the ion repeller of the reflectron MS. The deposition spot is $\sim 0.5 \mathrm{~mm}$ in diameter. A $10 \mathrm{~Hz}$ pulsed $266 \mathrm{~nm}$ laser (New Wave Research, Polaris III) is used to desorb and ionize the sample in situ. The laser irradiates the deposition spot with an incidence angle of 50 degrees (40 degrees from the horizontal plane), opposite to the particle beam. A quartz lens with $f=400 \mathrm{~mm}$ is used to softly focus the laser beam, also serving as a window. The laser spot is $\sim 0.2 \mathrm{~mm}$ in diameter. The ions generated by MALDI are detected with a reflectron mass spectrometer set at the vertical direction.

The three stage differential pumping system used to introduce the particles under atmospheric pressure into AL-MALDI MS is composed of three chambers: a source chamber, a differential chamber, and a detection chamber. An aperture with a diameter of $2 \mathrm{~mm}$ is used to connect the source chamber and the differential chamber, while another aperture with the same diameter is used to connect the differential chamber and the detection chamber. The distance between the exit of aerodynamic lens assembly and the deposition spot is $140 \mathrm{~mm}$. The source chamber is pumped by a $70 \mathrm{~L} \mathrm{~s}^{-1}$ rooster blower backed by an $8.3 \mathrm{~L} \mathrm{~s}^{-1}$ rotary pump. The differential chamber and detection chamber are pumped by a $320 \mathrm{~L} \mathrm{~s}^{-1}$ turbo pump individually. The two turbo pumps pumping the differential chamber and the detection chamber are backed by a $4 \mathrm{~L} \mathrm{~s}^{-1}$ rotary pump. The pressures in the source chamber and the detection chamber are $\sim 1 \mathrm{~Pa}$ and $\sim 5$ $\times 10^{-4} \mathrm{~Pa}$, respectively, during sampling.

The homemade reflectron mass spectrometer is characterized by a field-free flight distance of $1.2 \mathrm{~m}$, an ion mirror, and a chevron multichannel plate detector. The generated ions are extracted with a pulsed electric field ( $1200 \mathrm{~V}$ with a duration of $15 \mu \mathrm{s}$ ) of $550 \mathrm{~ns}$ after the laser shot. The metal ions from the target plate, salt ions,

(16) Zhang, X. F.; Smith, K. A.; Worsnop, D. R.; Jimenez, J.; Jayne, J. T.; Kolb, C. E. Aerosol Sci. Technol. 2002, 36 (5), 617-631. and matrix ions are gated by a time-delayed pulsed electric field ( $2100 \mathrm{~V}$ with a duration of $200 \mu \mathrm{s}$ ) applied to the ion mirror. The delay time for ion gating is changed from sample to sample to avoid saturation of the microchannel plates. The voltage for the acceleration field is $-8000 \mathrm{~V}$. The chevron microchannel plates float at $-8000 \mathrm{~V}$. The signal is amplified by a $20 \times$ amplifier (Ortec VT120C) and recorded with an oscilloscope (TEKTRONIX TDS3032B) which transfers data to a PC with an NI GPIB card.

The suspended particles for online MALDI analysis are generated by atomizing an analyte/matrix/salt-contained solution with a homemade continuous pneumatic atomizer. The solvent is $10 \%(\mathrm{~V} / \mathrm{V})$ water in isopropyl alcohol. The solution used in the experiment contains $0.1 \mu \mathrm{mol} \mathrm{mL} L^{-1}$ poly (ethylene glycol) (PEG, SCRC, China, chemical purity), $10 \mu \mathrm{mol} \mathrm{mL}^{-1} 2,5$-dihydroxybenoic acid (DHB, Sigma, 98\%), and $1 \mu \mathrm{mol} \mathrm{mL}^{-1} \mathrm{NaCl}$ (Beijing Shiji, China, 99.8\%), except for the special statements. The sizes of the generated particles are polydispersed with the diameters from tens of nanometers to hundreds of nanometers measured with a scanning mobility particle sizer (SMPS, TSI 3080). The mass concentration of PEG 1000 contained in the generated particles can be calculated with the formula of $C \cdot R_{1} \cdot M / R_{\mathrm{c}}$. $C$ is the concentration of PEG 1000 in the solution. $R_{1}$ is the liquid consumption rate of the solution, $\sim 0.003 \mathrm{~mL} \mathrm{~s}^{-1}$. $M$ is the molecular weight of PEG 1000. $R_{\mathrm{c}}$ is the flow rate of carrier gas for atomization, $25 \mathrm{~mL} \mathrm{~s}^{-1}$.

PEG is chosen as the sample in the test experiment due to its wide range of molecular weights. The polymers with the molecular weights from 200 to $20000 \mathrm{amu}$ are commercially available. The molecular structure of PEG can be represented as $\mathrm{HO}-\left(\mathrm{CH}_{2} \mathrm{CH}_{2}-\right.$ $O)_{n}-H$. In this paper, $\mathrm{PEG}_{\mathrm{n}}$ is used to represent the uniform PEG polymer with the degree of polymerization equal to $n$. Several papers reported the studies on the MALDI-TOF mass spectra of PEG and cationization of PEG molecules by alkali metal ions in MALDI. ${ }^{14,17}$ The MALDI mass spectra of PEG are obtained with AL-MALDI MS, indicating that the design can be used for online MALDI analysis.

\section{RESULTS AND DISCUSSION}

The PEG molecules are cationized by sodium ions in matrixassisted laser desorption/ionization. The mass spectra of PEG 1000 (A), 2000 (B), and 4000 (C) attached with sodium ions are shown in Figure 2. The numbers on the tops of the mass peaks indicate the degree of polymerization $(n)$. The strongest MALDI-TOF mass peaks of PEG 1000 and 2000 are almost consistent with the nominal degree of polymerization. However, the strongest MALDI-TOF mass peak of PEG 4000 appears around $m / z 3400$. The discrepancy may result from the nonuniform detection efficiency of the microchannel plates. The detection efficiency of the microchannel plates decreases quickly with the increase of the ion mass. ${ }^{18}$

Figure 3 shows MALDI-TOF mass spectra of $\left[\mathrm{PEG}_{22}+\mathrm{Na}\right]^{+}$ $(\mathrm{m} / z=1009.6)$ obtained with the ion extraction delayed at 550 ns after the laser shot. The delayed extraction is a widely used method to improve the mass resolution in MALDI-TOF mass spectrometry. ${ }^{19}$ The proper extraction delay can optimize

(17) Shimada, K.; Matsuyama, S.; Saito, T.; Kinugasa, S.; Nagahata, R.; Kawabata, S. Int. J. Mass Spectrom. 2005, 247 (1-3), 85-92.

(18) Fraser, G. W. Int. J. Mass Spectrom. 2002, 215 (1-3), 13-30.

(19) Brown, R. S.; Lennon, J. J. Anal. Chem. 1995, 67 (13), 1998-2003. 

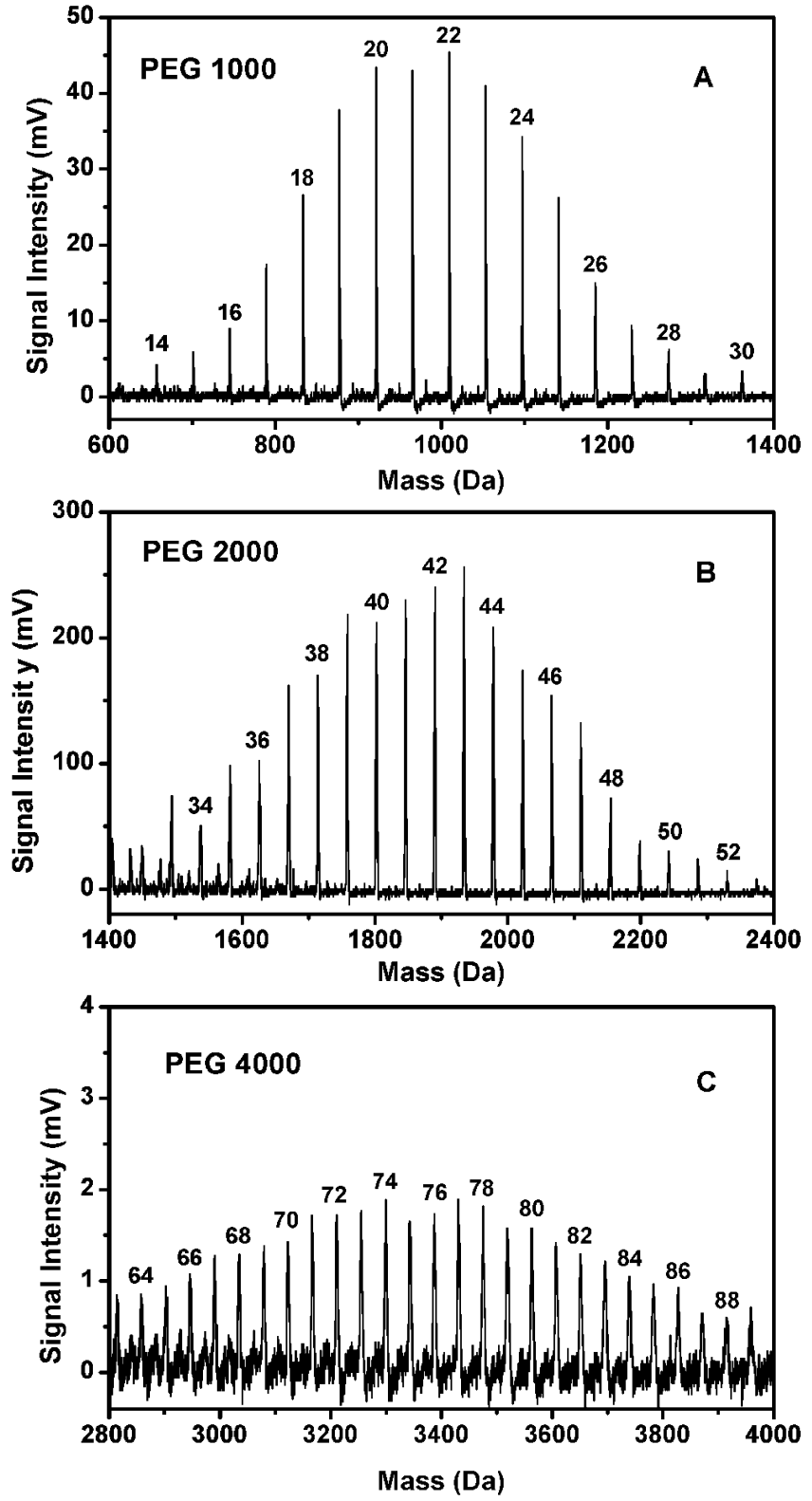

Figure 2. MALDI-TOF mass spectra of PEG 1000 (A), 2000 (B), and 4000 (C) attached with sodium ions. The ion extraction delay and ion gate delay are $550 \mathrm{~ns}$ and $16 \mu \mathrm{s}$ after laser irradiation. Each mass spectrum is acquired by averaging 128 pulses. The energy of the laser is $0.4 \mathrm{~mJ} /$ pulse.

time-of-flight compensation for ion energy spread. The mass spectrum obtained with $550 \mathrm{~ns}$ extraction delay presents the best mass resolution in the test experiment. The full width of the half maxium (fwhm) of the mass peak at 1009.6 Da is $\sim 0.5$ $\mathrm{Da}$. Therefore, the mass resolution of the mass spectrum is $\sim 2000 \mathrm{~m} / \Delta \mathrm{m}$. The mass resolution of the instrument is significantly affected by the laser energy, matrix, and analyte. The lower laser energy causes the better mass resolution. The mass spectra of the particles of polystyrene 1300/DHB/AgTFA (1:100:10) obtained under the similar experimental conditions present a much worse mass resolution $(\sim 400 \mathrm{~m} / \Delta \mathrm{m})$. These experimental results indicate that the size of the analyte ion pack is affected by the salt, matrix, and analyte.

The capability of the quantitative analysis of the instrument is investigated with the particles generated by atomizing four

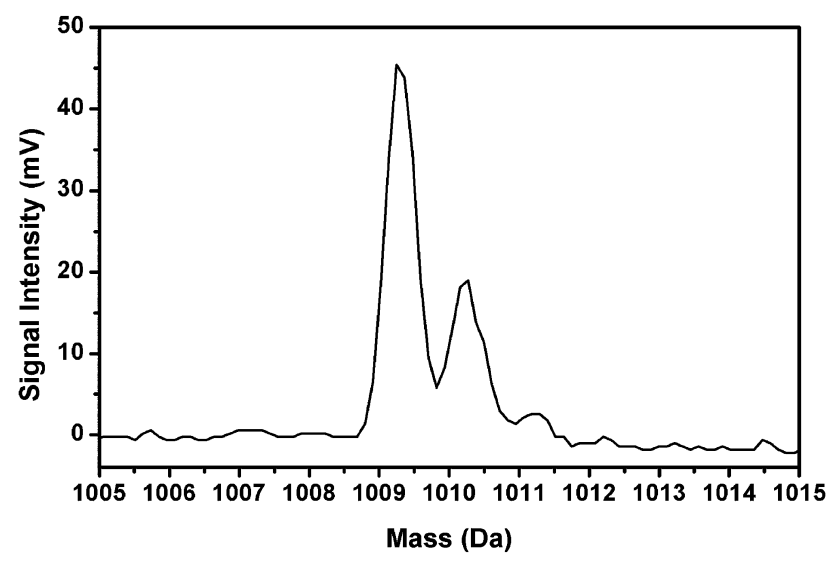

Figure 3. MALDI-TOF mass spectra of $\left[\mathrm{PEG}_{22}+\mathrm{Na}\right]^{+}$obtained with the ion extraction delay of $550 \mathrm{~ns}$ after laser irradiation. The mass spectra are acquired with an average of 128 laser pulses. The energy of the laser is $0.4 \mathrm{~mJ} /$ pulse.

Table 1. Mass Concentrations of the Generated Particles, Sampling Amounts of PEG $_{19}$, and Peak Intensities of $\left[\mathrm{PEG}_{\mathbf{1 9}}+\mathrm{Na}^{+}\right.$

$\begin{array}{lllll}C_{\mathrm{a}}\left(\mu \mathrm{mol} \mathrm{mL}^{-1}\right)^{a} & 0.04 & 0.12 & 0.20 & 0.28 \\ C_{\mathrm{b}}\left(\mu \mathrm{g} \mathrm{m}^{-3}\right) & 4.8 & 14.4 & 24.0 & 33.6 \\ Q_{\mathrm{a}}(\mathrm{pmol}) & 0.06 & 0.19 & 0.31 & 0.44 \\ Q_{\mathrm{b}}(\mathrm{pmol}) & 4 & 12 & 20 & 28 \\ S(\mathrm{mV}) & 42 \pm 7 & 422 \pm 19 & 752 \pm 60 & 1013 \pm 115\end{array}$

${ }^{a} C_{\mathrm{a}}, C_{\mathrm{b}}, Q_{\mathrm{a}}, Q_{\mathrm{b}}$, and $S$ in Table 1 stand for the concentrations of PEG 1000 in the solutions, the mass concentrations of the generated particles, the amounts of $\mathrm{PEG}_{19}$ deposited in $0.1 \mathrm{~s}$, the amounts of $\mathrm{PEG}_{19}$ deposited in $6.4 \mathrm{~s}$, and the signal intensity of $\left[\mathrm{PEG}_{19}+\mathrm{Na}\right]^{+}$, respectively. The standard deviations of the signal intensities are obtained from five measurements.

solutions with the concentrations of $0.04,0.12,0.20$, and $0.28 \mu \mathrm{mol}$ $\mathrm{mL}^{-1}$ PEG 1000 in water and isopropyl alcohol (1:9, V/V) solvent plus $10 \mu \mathrm{mol} \mathrm{mL}{ }^{-1} \mathrm{DHB}$ and $1 \mu \mathrm{mol} \mathrm{mL}{ }^{-1} \mathrm{NaCl}$. The corresponding mass concentrations of PEG 1000 contained in the particles generated with the four solutions are listed in Table 1. The MALDI-TOF mass spectrum of the particles generated with each solution is acquired. The ion extraction delay and ion gating delay are $550 \mathrm{~ns}$ and $18.3 \mu$ s after laser irradiation. The laser energy is $0.4 \mathrm{~mJ} /$ pulse. Each mass spectrum is acquired by averaging 64 laser pulses in $6.4 \mathrm{~s}$. The dependence of the signal intensity on the sampling amount of $\mathrm{PEG}_{19}$ is shown in Figure 4. The data points shown in Figure 4 are the mass peak intensities of $\left[\mathrm{PEG}_{19}+\mathrm{Na}\right]^{+}$, and their values are listed in Table 1 . Since the laser pulses irradiate the target plate at the repetition rate of $10 \mathrm{~Hz}$ during the experiment, the deposition time of the particles prior to each laser pulse is $0.1 \mathrm{~s}$. Without consideration of any possible residue, the amounts of particles deposited on the target plate can be calculated with the mass concentration of the generated particles and the sampling flow rate of AL-MALDI MS $\left(\sim 1.3 \mathrm{~mL} \mathrm{~s}^{-1}\right)$. Table 1 lists the deposition amounts of $\mathrm{PEG}_{19}$ in 0.1 and $6.4 \mathrm{~s}$. The abundance of $\mathrm{PEG}_{19}$ in the mixture of PEG 1000 is $\sim 0.10$, estimated according to the mass spectrum shown in Figure 2A. Since the noise level of the instrument is $\sim 1 \mathrm{mV}$ and the signal intensity of $\mathrm{PEG}_{19}$ with the sampling amount of 4 pmol is $42 \mathrm{mV}$, the detection limit of the instrument for PEG is $\sim 0.3 \mathrm{pmol}$, calculated with 3 times of the noise level. 


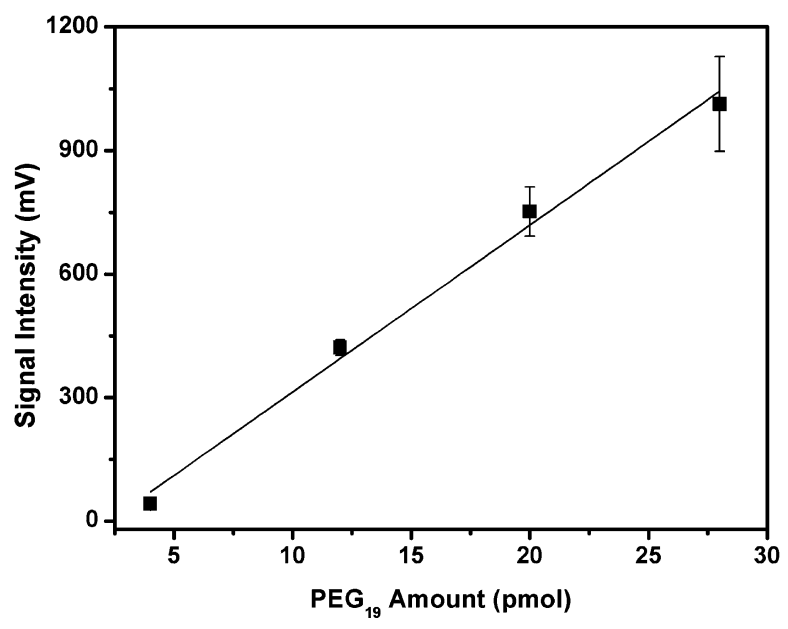

Figure 4. Dependence of the signal intensity on the sampling amount of $\mathrm{PEG}_{19}$. The error bars are the standard deviation of five measurements. The datum point at $28 \mathrm{pmol}$ is measured at a lower MCP voltage to avoid the overload of the MCP and is normalized to the value of the datum point at 20 pmol.

Figure $4 \mathrm{~L}$ reveals that the signal intensity of $\left[\mathrm{PEG}_{19}+\mathrm{Na}\right]^{+}$ is in a linear correlation with the sampling amount of $\mathrm{PEG}_{19}$ with the correlation coefficient $\left(R^{2}\right)$ of 0.99 . The slope is 40.5 $\pm 2.4 \mathrm{mV} / \mathrm{pmol}$. The linear correlation between the amount of the sample and its signal only remains in a small range from 4 to $28 \mathrm{pmol}$. The nonlinear correlation is observed when the sampling amount is beyond the range. The nonlinear correlation may partially result from the characterization of MALDI and partially from the capability of the instrument (such as saturation of $\mathrm{MCP}$ ). We try to get the dependence of the signal intensity on the sampling amount of PEG with a fixed ratio between PEG and DHB/ $\mathrm{NaCl}$. However, the dependence has not been obtained due to the too large uncertainty.

The aerodynamic lens assembly has been used as the particle inlet system for aerosol mass spectrometers ${ }^{20}$ and aerosol-MALDI MS. ${ }^{13,14}$ Gao and Johnston used an aerodynamic lens assembly to prepare the sample on the target plate for MALDI analysis. ${ }^{15}$ AL-MALDI MS utilizes the aerodynamic lens assembly to transport the suspended sample particles directly onto the target plate and conduct in situ MALDI analysis. The efficiency of sample delivery of aerosol-MALDI MS is limited by the repetition rate of the laser and most of the sampled particles may fly through the detection region without hitting the nanosecond desorption/ionization laser pulse. Compared with aerosol-MALDI MS, AL-MALDI MS is a bulk technique. It possesses the potentially better efficiency of sample delivery when single-particle information is not required by analysis. Additionally, the signal intensity of the single laser pulse can be controlled by chopping laser pulses to change the deposition time of the sample. Most of the analyte (PEG) deposited inside the laser spot during the dwelling time of the

(20) Jayne, J. T.; Leard, D. C.; Zhang, X. F.; Davidovits, P.; Smith, K. A.; Kolb, C. E.; Worsnop, D. R. Aerosol Sci. Technol. 2000, 33 (1-2), 49-70. laser is consumed by the following laser pulse when the instrument samples the PEG particles generated from the solution with the concentration of PEG lower than $0.3 \mu \mathrm{mol} \mathrm{mL} \mathrm{m}^{-1}$. The sample accumulated outside the laser spot does not apparently affect the PEG signal. The signal of PEG disappears in a short time after sampling ceases. However, the small ion signal of [PEG $+\mathrm{Na}^{+}$is observed $10 \mathrm{~min}$ after the sample of $\mathrm{PEG} / \mathrm{NaCl}$ / DHB is switched to the sample of $\mathrm{PEG} / \mathrm{KCl} / \mathrm{DHB}$, which indicates that the salt is not consumed by the laser pulse instantly.

\section{CONCLUSIONS}

The MALDI-TOF mass spectra of PEG 1000, 2000, and 4000 attached with sodium ions are obtained in the experiment. The mass resolution of the instrument reaches $2000 \mathrm{~m} / \Delta \mathrm{m}$. The signal intensity of $\left[\mathrm{PEG}_{19}+\mathrm{Na}\right]^{+}$is in a linear correlation with the sampling amount of $\mathrm{PEG}_{19}$ when the sampling amount is in the range from 4 to $28 \mathrm{pmol}$. These initial experimental results demonstrate that AL-MALDI MS is capable of conducting in situ MALDI analysis and using an aerodynamic lens assembly as a direct deposition interface provides an alternative method for online MALDI analysis. The main disadvantage of this design is the residue of the sample on the target plate. The contamination of the residual sample on the target plate might be tolerated if the instrument is used to online monitor some analytes with the same matrix and salt. However, the setting of the target plate must be improved if the instrument is used to analyze different chemicals with different matrixes like classic MALDI MS. Recently, the issue of bioaerosols has caused a great concern. The technique of aerosol-MALDI MS has been tested as a method to offer online monitoring of bioaerosols. ${ }^{21,22}$ Limited by the repetition rate of the laser, some of the bioaerosol particles sampled by aerosol-MALDI MS may miss detection. The motivation to develop this instrument is to offer an alternative approach for the online analysis of bioaerosols and AL-MALDI MS may have the potential to obtain more comprehensive mass spectrometric information of bioaerosols.

\section{ACKNOWLEDGMENT}

This research was funded by National Natural Science Foundation of China (Grant No. 20777082 and 50921064) and National High Technology Research and Development Program of China (863 Program, Grant No. 2009AA06Z401).

Received for review April 9, 2010. Accepted May 26, 2010.

\section{AC100941Z}

(21) van Wuijckhuijse, A. L.; Stowers, M. A.; Kleefsman, W. A.; van Baar, B. L. M.; Kientz, C. E.; Marijnissen, J. C. M. J. Aerosol Sci. 2005, 36 (5-6), 677687.

(22) McJimpsey, E. L.; Jackson, W. M.; Lebrilla, C. B.; Tobias, H.; Bogan, M. J.; Gard, E. E.; Frank, M.; Steele, P. T. J. Am. Soc. Mass Spectrom. 2008, 19 (3), 315-324. 